\title{
Three-Year Outcomes of Implantable Collamer Lens Followed by Excimer Laser Enhancement ("Bioptics") in the Treatment of High Myopic Astigmatism
}

This article was published in the following Dove Press journal:

Clinical Ophthalmology

Samir Jabbour
Kraig S Bower

The Wilmer Eye Institute, Johns Hopkins University School of Medicine, Baltimore, MD, USA
Correspondence: Samir Jabbour

The Wilmer Eye Institute, Johns Hopkins School of Medicine, 600 North Wolfe

Street, Baltimore, MD, 21287, USA

Tel + I 4l0 583-2843

Email samir.jabbour2@gmail.com
Purpose: Describe three-year outcomes of spherical implantable Collamer lens (ICL) followed by excimer laser enhancement (bioptics) in eyes with high myopic astigmatism.

Patients and Methods: Retrospective case series of thirty-four cases that underwent bioptics enhancement at the Johns Hopkins Wilmer Eye Institute. All eyes had a preoperative sphere of $-6.00 \mathrm{D}$ or more with a cylinder of at least $2.00 \mathrm{D}$. Uncorrected and corrected distance visual acuity (UDVA and CDVA), manifest spherical equivalent refraction (MSE), ICL vault measurements and central corneal thickness (CCT) were collected. Endothelial cell counts (ECC), root mean square (RMS) of higher order aberrations (HOAs), adverse events and subsequent surgeries were also assessed.

Results: All patients had a minimum follow-up of 3 years. Preoperative UDVA was $2.29 \pm$ $0.46 \log$ MAR and improved to $0.03 \pm 0.23 \log$ MAR at 3 years $(\mathrm{p}<0.05)$. MSE was $-12.30 \pm$ 4.05 preoperatively and changed to $-0.21 \pm 0.46$ at 3 years $(\mathrm{p}<0.05)$. The efficacy and safety indices were $1.28 \pm 0.32$ and $1.47 \pm 0.27$ at 3 years post-enhancement. HOA did not significantly change throughout the follow-up $(\mathrm{p}<0.05)$. Endothelial cell loss at 12 months was calculated at $5.7 \%$. Two eyes required ICL exchange due to vault-related issues.

Conclusion: Bioptics offered excellent long-term safe, predictable, and efficient outcomes for high myopic astigmatism and can be considered an option if toric ICL is not available. Results confirm that wavefront-guided photoablation remains an excellent option to manage residual refractive error after phakic IOL.

Keywords: bioptics, implantable collamer lens, enhancement, excimer laser

\section{Introduction}

Full correction of high degrees of myopia or myopic astigmatism is seldom possible with corneal-based refractive procedures such as laser-assisted in-situ keratomileusis (LASIK) or photorefractive keratectomy (PRK). High degrees of tissue ablation with these procedures would alter the biomechanics of the cornea and significantly increase the risk of ectasia. ${ }^{1-3}$ Higher order aberrations (HOAs), night glare and halos could also ensue., ${ }^{4,5}$

Since their approval two decades ago, refractive surgeons have since opted to the use phakic intraocular implants to treat high myopia not amenable to excimer laser surgery. The Visian Implantable Collamer Lens (ICL) (STAAR Surgical, Monrovia, California, USA), a posterior chamber phakic intraocular lens (IOL) 
that was FDA approved in 2005, has been shown to be effective in the correction of myopia up to $-18.00 \mathrm{D}^{6-9}$ Up until 2018, only the spherical model of the ICL was available in the USA. As such, high myopia and high astigmatism could not be corrected with a single procedure. Some refractive surgeons consequently adapted to a consecutive procedure strategy consisting of ICL implantation followed by an excimer-laser enhancement. This sequence has been referred to as "bioptics". 10-13

While this approach has been used by many refractive surgeons, there is relatively few published data on longterm reports of outcomes of bioptics in patients with myopic astigmatism treated in the USA. With the recent approval of the toric ICL (TICL), large amounts of myopia and astigmatism can be corrected safely and effectively in a single procedure, and a two-step bioptics approach is not necessary. ${ }^{14-16}$ Nevertheless, reporting bioptics outcomes could be used as a reference for future comparative studies in this patient population. Furthermore, refractive surprises are possible with TICL, requiring excimer laser enhancements, similar to bioptics. In this retrospective study, we describe our single-center 3-year outcomes of bioptics for patients with high myopic astigmatism.

\section{Methods}

\section{Study Design}

This retrospective study was performed at the Johns Hopkins Wilmer Eye Institute in Baltimore, Maryland and conducted in accordance with the tenets of the Declaration of Helsinki. The study was approved by the Johns Hopkins Hospital Institutional Review Board reference. The medical record of 17 patients (34 eyes) treated between October 2011 and August 2020 were included in the analysis. Informed consent of the study participants was waived due to the retrospective nature of the study.

All patients who underwent ICL implantation followed by excimer laser ablation with PRK or LASIK were reviewed. To be included in the study, patients had to have at preoperative sphere of $-6.00 \mathrm{D}$ or more with a cylinder of at least $2.00 \mathrm{D}$ in one or both eyes. Patients with prior corneal or intraocular surgery or other ocular morbidities that could influence postoperative parameters were excluded. Patients with less than 3 years follow-up from the time of enhancement were excluded from analysis.

All participants underwent a comprehensive ophthalmologic examination preoperatively including uncorrected distance visual acuity (UDVA), corrected distance visual acuity (CDVA), manifest refraction, slit lamp examination, intraocular pressure, and dilated fundus examination. Visual acuity was assessed using a backlit Snellen chart with a test distance equivalent to 6-m. Additional testing included corneal topography, central corneal thickness (CCT), and anterior chamber depth (ACD) measured by Pentacam $^{\mathrm{TM}}$ (Oculus, Arlington, VA, USA), endothelial cell counts (ECC) measured with specular microscopy (Specular Microscope CEM-530, Nidek, Gamagori, Aichi, Japan), root mean square (RMS) of higher order aberrations (HOAs) measured with the WaveScan WaveFront System (J\&J Surgical Vision, Santa Ana, CA), and anterior segment optical coherence tomography (AS-OCT) (Visante, Carl Zeiss Meditec, Oberkochen, Germany). Patients with history or evidence of glaucoma, iritis, pigment dispersion, autoimmune disorder, pregnancy, other ocular or systemic contraindication, those with inadequate $\mathrm{ACD}(<3.0 \mathrm{~mm})$ or irregular, and those with abnormal/suspicious corneal topography were excluded from treatment. All patients with posterior segment pathology were referred for retinal consultation preoperatively.

Data was collected preoperatively, at three months postoperatively following ICL implantation, and at the 1and 3-years following enhancement. Any intraoperative or postoperative complications were noted throughout the follow-up period.

The primary outcome measures were the UDVA, CDVA, MSE at the 3-year post-enhancement visit. Secondary outcome measures included endothelial cell density and wavefront aberrometry at the 12-months visit, vault measurements over time, and keratometry and visual outcomes at the 3 years visit. Any intraoperative and postoperative complications or adverse effects were noted, as well as need for any additional surgeries.

\section{Visian ICL Surgical Procedure}

The size and power of the ICL was determined using the Online Calculation and Order System (OCOS) nomogram (https://ocos.staarag.ch/). The power was determined based on the patient's manifest refraction, and the diameter of the lens on biometric data that included anterior chamber depth and white-to-white measurements from optical biometry (IOLMaster 500, Carl Zeiss Meditec, Oberkochen, Germany) and Scheimpflug imaging measurements (Pentacam, Oculus, Arlington, VA). All eyes 
were targeted for postoperative spherical equivalent of plano.

Each patient underwent two peripheral iridotomies per eye at 10:30 and 1:30 using Nd:YAG laser at least 2 weeks prior to surgery. Under monitored anesthesia care and topical anesthesia, a 15-degree super blade was used to create a paracentesis at the limbus. OcuCoat (Bausch \& Lomb, Laval, QC, Canada) viscoelastic was then used to fill the anterior chamber. A 3.0-mm keratome was used to create a clear corneal temporal incision. The implant was then loaded in the standard cartridge and injected through the main corneal incision. A Batlle manipulator (Diamatrix Inc., Bucks County, PA, USA) was used to position the four footplates of the ICL under the iris taking caution not to touch the ICL optic, crystalline lens or corneal endothelium. Once the plates were positioned behind the pupil, the viscoelastic was gently irrigated and aspirated from the anterior chamber and the pupil was constricted using intracameral Miochol (Bausch \& Lomb, Laval, QC, Canada). At the end of the case, the wounds were hydrated with balanced salt solution (BSS).

Patients were treated with moxifloxacin $0.5 \%$ drops four times daily for one week and prednisolone acetate $1 \%$ drops four times a day for one week then twice a day for 3 weeks. Patients were seen postoperatively one day, one week, and then one, two and three months post-ICL. By the three-month postoperative examination, the patient was considered eligible for excimer enhancement if the refraction, Wavescan and topography were stable. Patients who elected to wait for enhancement were followed every six months until enhancement procedure.

\section{Excimer Laser Enhancement (LASIK, PRK)}

All photoablation procedures (LASIK and PRK) were wavefront-guided CustomVue ${ }^{\mathrm{TM}}$ ablations performed with the VISX Star S4 IR Active Trak platform (J\&J Surgical Vision, Santa Ana, CA, USA) (software version 5.32). Treatment planning was determined from wavefront aberrometry captured and calculated using the WaveScan wavefront analyzer (Johnson \& Johnson Vision Care, Inc., Santa Ana, California, USA) and targeting emmetropia.

LASIK flap creation was done with the IntraLase FS Laser platform (J\&J Surgical Vision, Santa Ana, CA, USA) with a $9.2 \mathrm{~mm}$ diameter and a $120 \mathrm{~mm}$ thickness with a superior hinge. Postoperatively patients were treated with moxifloxacin $0.5 \%$ drops four times a day for one week, prednisolone acetate 1\% drops every hour for 24 hours then four times a day for one week and preservativefree carboxymethylcellulose $0.5 \%$ every hour for the first week then at least every 2 hours or more for several months. Patients were seen one day and one week postoperatively to assess for early healing complications.

For all PRK procedures, the corneal epithelium was exposed to ethanol $20 \%$ for 30 seconds and then removed with a Weck-Cel spear (BVI Medical, Waltham, MA, USA). Once excimer laser was applied, mitomycin C $0.2 \mathrm{mg} / \mathrm{mL}(0.02 \%)$ was placed on the stroma for 30 seconds and then irrigated with chilled BSS. At the end of the procedure, a bandage contact lens (Acuvue Oasys, Johnson \& Johnson Vision Care), was placed on the eye Patients were treated with moxifloxacin $0.5 \%$ drops four times a day for one week, fluorometholone $0.1 \%$ ophthalmic suspension four times a day for one month, then tapered over the ensuing six weeks (three times a day for 2 weeks, then twice a day for 2 weeks, then once a day for 2 weeks), and frequent preservative-free carboxymethylcellulose $0.5 \%$ at least every 2 hours or more for several months. Patients were seen on postoperative day one and a second early postoperative visit on day four to seven for contact lens removal.

\section{Statistical Analysis}

Results are expressed as mean \pm standard deviation (range) and were calculated using Microsoft Excel 2019 (Microsoft, Redmond, WA, USA). All statistical analyses were performed using SPSS v25.0 (IBM corporation, Armonk, New York, USA). Snellen visual acuity was converted to logarithm of the minimum angle of resolution $(\log M A R)$ equivalents for statistical analysis. Visual acuity of a hand motion was set at $2.3 \log$ MAR and counting fingers at $1.85 \log$ MAR. A Student's $t$-test and a Wilcoxon signed rank test were used for statistical analysis to compare pre- and post-treatment variables depending on data distribution. The Efficacy Index was defined as UDVA after treatment divided by CDVA before treatment (UDVA post/CDVA pre). The Safety Index was defined as CDVA after treatment divided by CDVA before treatment (CDVA post/CDVA pre). A vectorial analysis was performed using the ISRS calculator (https://www.isrs.org/ resources/assort-group-analysis-calculator), and followed the guidelines of the Journal of Refractive Surgery report of astigmatism outcomes. ${ }^{17}$ A value of $p<0.05$ was considered statistically significant. 


\section{Results}

In this study, ICL implantation followed by excimer laser enhancement was performed in a total of 34 eyes of 17 patients ( 8 males and 9 females). Mean age was $30.0 \pm 6.9$ years (21.0 to 45.0$)$. No patients were lost to follow-up at the minimal 3-year postoperative period following laser enhancement. Table 1 summarizes the patient's preoperative and postoperative refractive characteristics.

The average time between ICL implantation and excimer laser enhancement was $172.0 \pm 127.1$ days $(87.0$ to 557.0 days). Twenty-eight wavefront-guided PRK and 6 wavefront-guided LASIK treatments were performed for enhancement. Iris Registration (IR) was obtained in 30 of 34 eyes $(88.2 \%)$.

\section{Refractive Outcomes}

Preoperative UDVA was $2.29 \pm 0.46 \log$ MAR (equivalent to 20/400 and worse) prior to surgery. At the 3-year postoperative visit, UDVA improved to $0.03 \pm 0.23 \log$ MAR $(\mathrm{p}<0.05)$. Compared to preoperative CDVA (logMAR 0.08 \pm 0.11 ), final postoperative CDVA showed a trend towards improvement $(\log$ MAR $-0.06 \pm 0.69)$ but this was not statistically significant $(\mathrm{p}>0.05)$. MSE was $-12.30 \pm 4.05$ $\mathrm{D}$ preoperatively and decreased to $-0.21 \pm 0.46 \mathrm{D}$ at the 3 -year visit $(p<0.05)$ (Table 1). After enhancement, all eyes gained 1-3 lines of CDVA.

Refractive outcomes at the 3-year post-enhancement visit are illustrated in Figure 1 following the Standard Graphs for Reporting Refractive Surgery:
- Efficacy. Eighty-five percent (85\%) of eyes achieved UDVA of $20 / 20$ or better and $38 \%$ achieved 20/16 and better at the 3-year postoperative period (Figure 1A). The mean Efficacy Index at 3-year postenhancement was $1.28 \pm 0.32$ (1.00 to 2.00).

- Safety. CDVA was favorably maintained throughout the follow-up period. Compared to best-corrected preoperative line, no eyes lost any line at the 3-year follow-up with a mean Safety Index of $1.47 \pm 0.27$ (1.25 to 2.67) (Figure 1B).

- Attempted versus Achieved Spherical Equivalent. The coefficient of determination (R) between attempted SE and achieved SE was 0.97. The mean achieved SE was $-12.30 \pm 4.05 \mathrm{D}(-7.75$ to -19.88$)$ (Figure 1C). Two eyes were overcorrected and one eye was under corrected. There were no identifiable causes that explained these refractive surprises.

- Predictability. At 3-years, 89\% of eyes achieved MSR within $0.50 \mathrm{D}$ of emmetropia of the attempted SE correction (Figure 1D).

- Refractive Astigmatism. Keratometric cylinder decreased from $2.24 \pm 0.70 \mathrm{D}$ (1.10 to 3.70) preoperatively to $0.87 \pm 0.39 \mathrm{D}(0.20$ to 1.30$)$ at 3 -year post-enhancement $(\mathrm{p}<0.05)$. The mean preenhancement residual refractive astigmatism was $2.36 \pm 0.72 \mathrm{D}(+1.00$ to 4.00$)$. Mean refractive astigmatism significantly decreased at the 3 -year period to $0.33 \pm 0.34 \mathrm{D}(0.00$ to 1.25$)$ with $88 \%$ of eyes having a residual astigmatism of less than $0.50 \mathrm{D}(\mathrm{p}<0.05)$ (Figure 1E). Figure 2 shows the astigmatism

Table I Preoperative and Postoperative Clinical Outcomes of 34 Eyes from 17 Patients

\begin{tabular}{|c|c|c|c|c|}
\hline \multirow[t]{2}{*}{ Characteristics } & \multicolumn{4}{|l|}{ Mean \pm Standard Deviation } \\
\hline & Preop & 3-Month Post-ICL & $\begin{array}{l}\text { I2-Month Post- } \\
\text { Enhancement }\end{array}$ & 3-Years Post-Enhancement \\
\hline UDVA, LogMAR & $2.29 \pm 0.46(2.00$ to 3.00$)$ & $0.34 \pm 0.29(0.00$ to 1.00$)$ & $-0.02 \pm 0.15(-0.12$ to 0.30$)$ & $0.02 \pm 0.16(-0.12$ to 0.70$)(p=0.13)$ \\
\hline CDVA, LogMAR & $0.08 \pm 0.11(0.00$ to 0.40$)$ & $-0.02 \pm 0.08(-0.12$ to 0.18$)$ & $-0.06 \pm 0.07(-0.12$ to 0.10$)$ & $-0.06 \pm 0.69(-0.10$ to 0.10$)(p=0.09)$ \\
\hline MSE, D & $-12.30 \pm 4.05(-19.88$ to -7.75$)$ & $-0.51 \pm 1.12 \mathrm{D}(-4.38$ to 0.75$)$ & $-0.09 \pm 0.36(-1.00$ to 0.63$)$ & $-0.21 \pm 0.46(-1.63$ to 0.63$)(p<0.05)$ \\
\hline Manifest cylinder, D & $2.63 \pm 0.73(1.50$ to 4.25$)$ & $2.17 \pm 0.72(1.00$ to 4.00$)$ & $-0.09 \pm 0.36(-1.00$ to 0.63$)$ & $0.34 \pm 0.40(0.00$ to 1.75$)(p<0.05)$ \\
\hline Average $\mathrm{K}, \mathrm{D}$ & $44.40 \pm 1.29(40.25$ to 46.15$)$ & $44.27 \pm 1.18(41.30$ to 47.20$)$ & $43.40 \pm 2.12(36.15$ to 46.80$)$ & $43.36 \pm 2.05(36.25$ to 46.55$)(p=0.46)$ \\
\hline Cylindrical K, D & $2.24 \pm 0.71(1.10$ to 3.70$)$ & $2.35 \pm 0.69(0.60$ to 3.70$)$ & $0.74 \pm 0.33(0.20$ to 1.50$)$ & $0.87 \pm 0.40(0.10$ to 2.00$)(p=0.34)$ \\
\hline $\mathrm{ECD}\left(\mathrm{c} / \mathrm{mm}^{2}\right)$ & $3046 \pm 212(2774$ to 3526$)$ & $2894 \pm 246(2178$ to 3466$)$ & $2871 \pm 312(2062$ to 3485$)$ & N/A \\
\hline CCT (um) & $532.2 \pm 24.3$ (496 to 589$)$ & $539.5 \pm 26.2(499$ to 593$)$ & $505.8 \pm 28.6(462$ to 551$)$ & $511.5 \pm 25.6(455$ to 558$)(\mathrm{p}=0.19)$ \\
\hline HOA RMS (um) & $0.28 \pm 0.10(0.12$ to 0.49$)$ & $0.29 \pm 0.1(0.15$ to 0.51$)$ & $0.37 \pm 0.10(0.20$ to 0.66$)$ & N/A \\
\hline Vault (um) & N/A & $567 \pm 207(200$ to 1120$)$ & $469 \pm 195(80$ to 940$)$ & $431 \pm 190(80$ to 880$)(p=0.18)$ \\
\hline
\end{tabular}

Note: $\mathrm{p}$-values are calculated between 12 months and 3 years post enhancement.

Abbreviations: ICL, implantable collamer lens; UDVA, uncorrected distance visual acuity; CDVA, corrected distance visual acuity; MSE, manifest spherical equivalent; K, keratometry; ECD, endothelial cell density; CCT, central corneal thickness; HOA RMS, higher order aberration root mean square. 


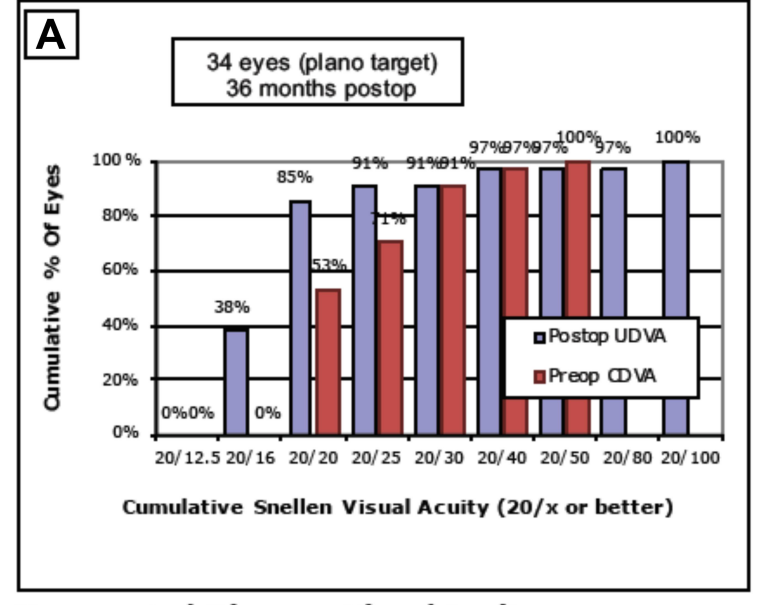

Uncorrected Distance Visual Acuity

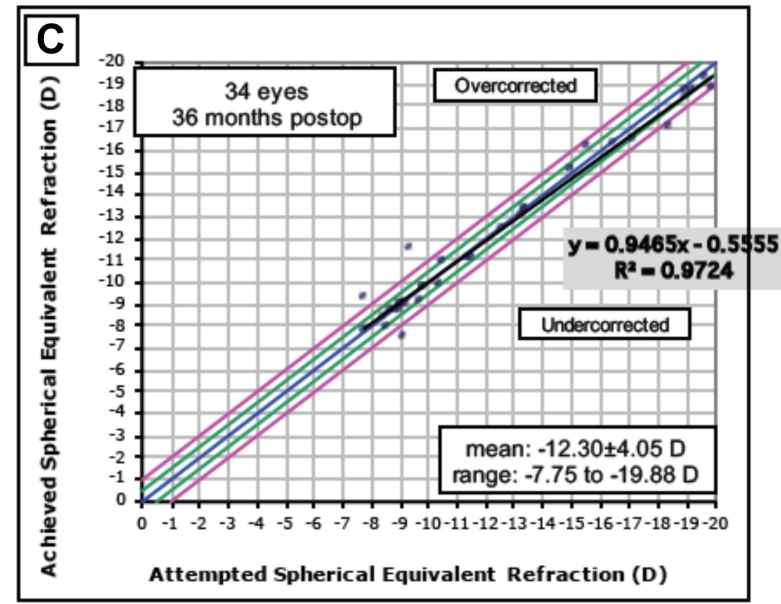

Spherical Equivalent Attempted vs Achieved

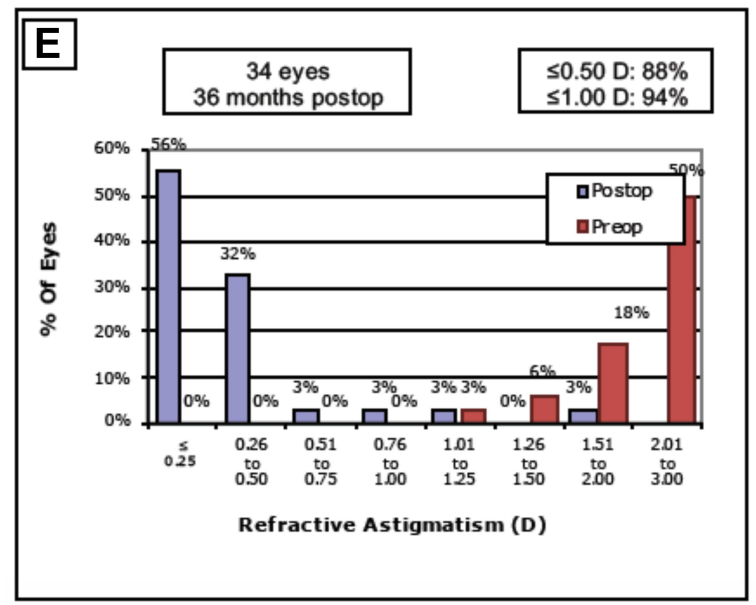

Refractive Astigmatism

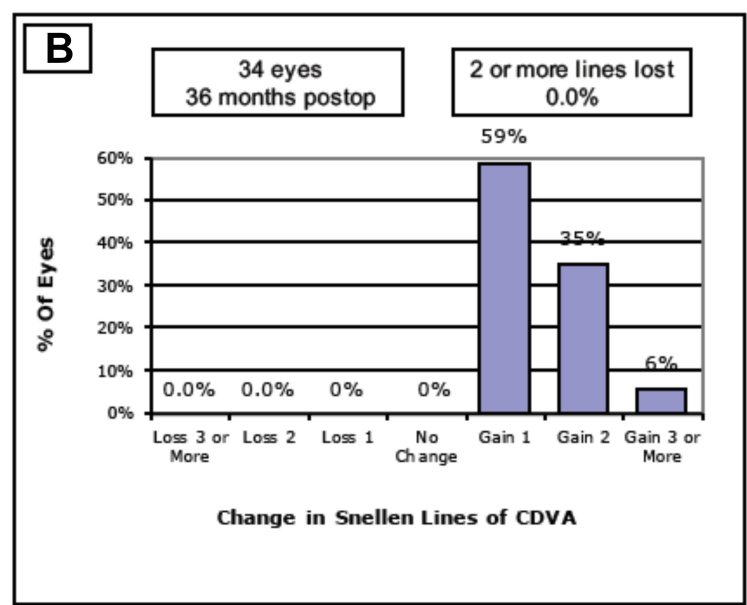

Change in Corrected Distance Visual Acuity

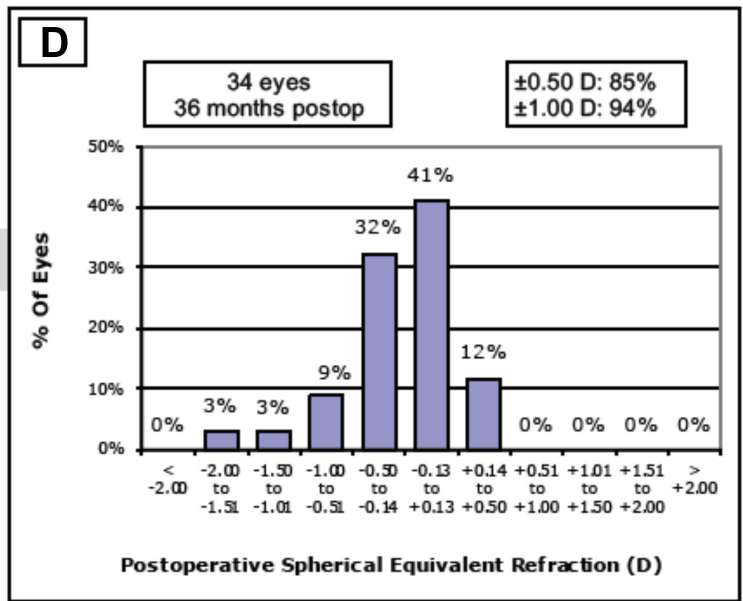

Spherical Equivalent Refractive Accuracy

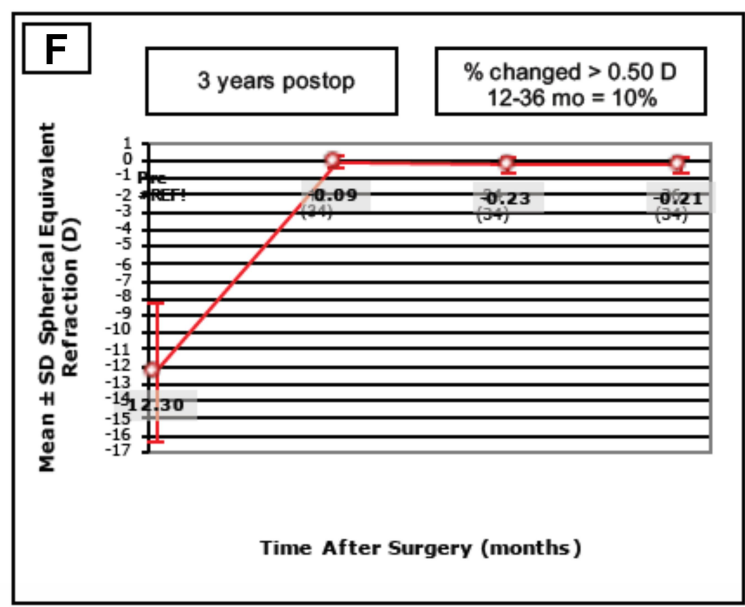

Stability of Spherical Equivalent Refraction

Figure I Standard graphs for reporting refractive surgery. (A) Distribution of uncorrected distance visual acuity (UDVA) at 3-year against distribution of preoperative corrective distance visual acuity (CDVA). (B) Change in corrected distance visual acuity (CDVA) from baseline at 3-year postoperatively. (C) Refractive accuracy attempted versus achieved spherical equivalent refraction. (D) Distribution of manifest refraction (MSE) at 3-year postoperatively. (E) Comparison of refractive astigmatism preoperatively and at 3-year postoperatively. (F) Mean manifest refraction (MSE) from pre-enhancement up to 3 years postoperatively. 
vectorial analysis graphs. The overall difference vector was $0.17 \mathrm{D}$ at axis 17 and the overall corrective index (surgically induced astigmatism/target induced astigmatism) was 0.92 .

- Stability. Figure 1F shows the achieved MSE as a function time, up to 3 years post-enhancement. MSE remained relatively stable and close to emmetropia throughout the follow-up period. Between 12 months and 3 years, $10 \%$ had fluctuation greater than $0.50 \mathrm{D}$.

\section{Endothelial Cell Density and Central Corneal Thickness}

The mean preoperative ECD was $3046 \pm 212$ cells $/ \mathrm{mm}^{2}$ and decreased to $2871 \pm 312$ cells $/ \mathrm{mm} 2$ (2062 to 3485 ) at 12 months post-enhancement $(\mathrm{p}<0.05)$. The mean percentage of endothelial cell loss was $5.7 \%$ at 12 months postenhancement. CCT remained stable at 3-year post enhancement ( $p>0.05)$ (Table 1).
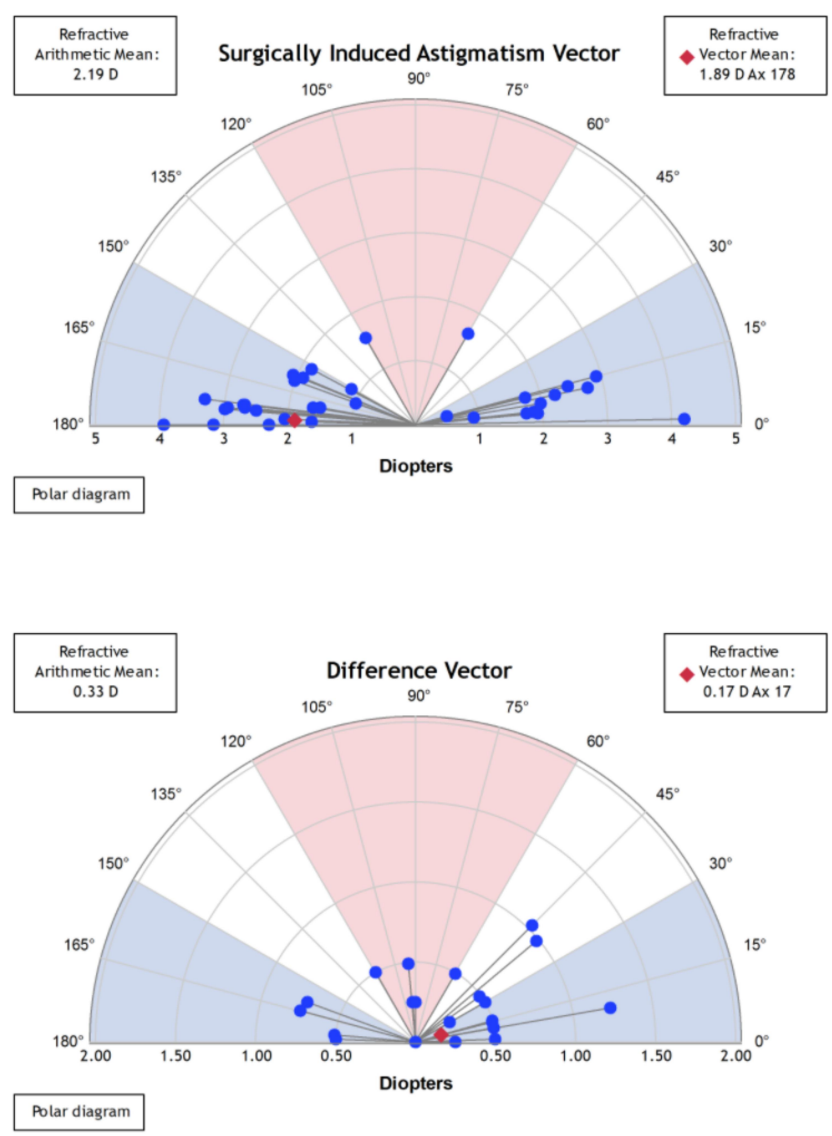

\section{Vault Measurements}

The vault measured with AS-OCT decreased over time but this change was not found to be statistically significant at 3-year post-enhancement (Table 1).

\section{Higher Order Aberrations}

The RMS of total HOAs was slightly increased at 12-month post-enhancement but was not statistically significant ( $p>0.05$ ) (Table 1). Six eyes developed mild night glare following enhancement and were started on brimonidine drops with improvement in symptoms. In these patients, preoperative HOA was $0.31 \pm 0.07$ um (0.28 to $0.46)$ and increased to $0.42 \pm 0.18$ um $(0.20$ to 0.66$)$ $(\mathrm{p}<0.05)$, and their mean post-enhancement residual astigmatism was $0.25 \mathrm{D}$ ( 0.00 to 0.50$)$.

\section{Secondary Surgeries and Adverse Events}

There were no intraoperative complications. One eye was found to have a steep vault, and one eye had a shallow
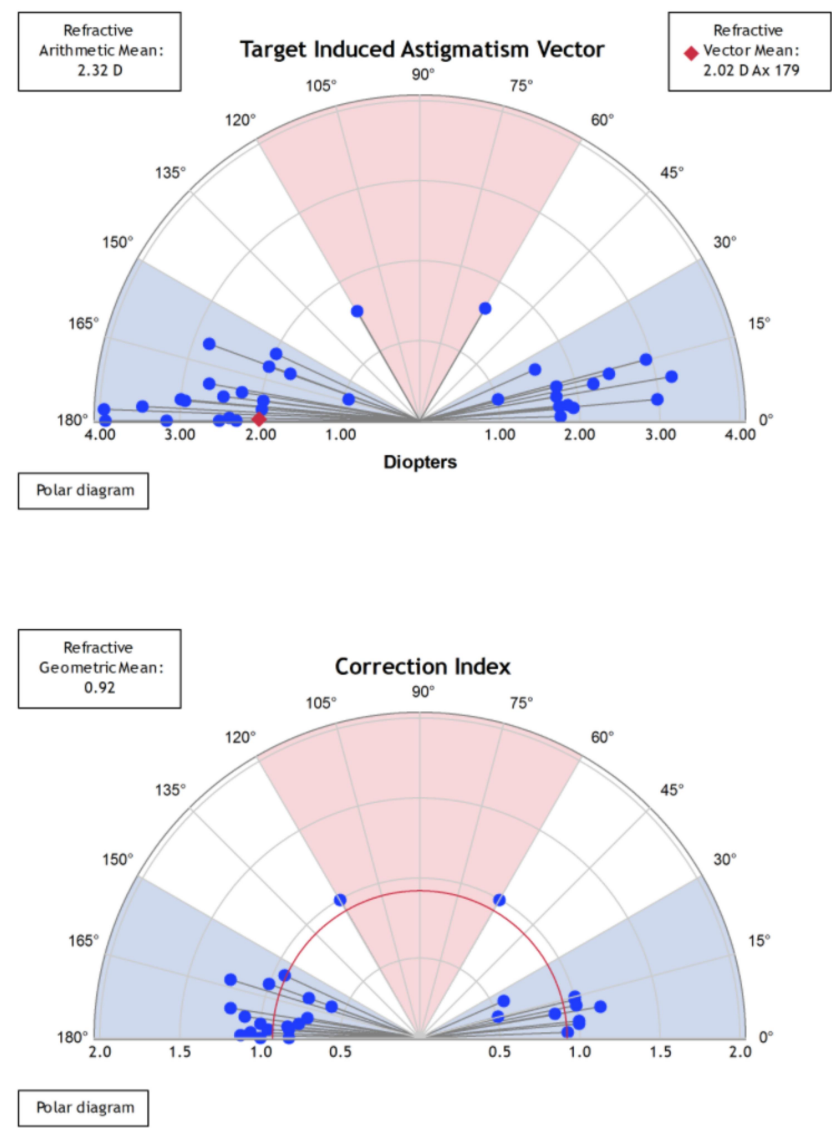

Figure 2 Overview of Alpins vectorial analysis of corneal astigmatism following laser enhancement surgery at 3-year postoperatively. The target-induced astigmatism (TIA) shows the range of astigmatism that the surgery intended to induce. The surgically induced astigmatism (SIA) shows the ranged of achieved astigmatism. The difference vector (DV), calculated as SIA minus TIA, shows the remaining astigmatism following enhancement. The correction index (CI), calculated as SIA divided by TIA, shows the under/overcorrection of the astigmatism treatment, and can be used as a measure of success with an optimal value close to I. 
vault that both warranted ICL exchange. Three patients developed trace anterior subcapsular cataract that did not affect vision and were observed. Two eyes developed glaucoma secondary to steroid response that were adequately controlled with topical drops. Intraocular pressure returned to baseline on no drops after completing topical steroid taper. One eye developed a rhegmatogenous retinal detachment three years after surgery that was treated with a scleral buckle with resulting myopic refraction of -2.00 D. Pigment dispersion glaucoma, iritis or pupillary block were not observed in the study.

\section{Discussion}

In this study, we report outcomes of spherical ICL implantation followed by wavefront-guided excimer laser enhancement with LASIK or MMC-PRK ("bioptics") for high myopic astigmatism. We demonstrate that bioptics is effective, predictable and safe in the treatment of high myopic astigmatism (sphere $<-6.00 \mathrm{D}$ and cylinder $>2.00 \mathrm{D}$ ) up to 3 years postoperatively. Despite the retrospective nature of this study and relatively small sample size, data was complete for all patients up to the 3-year post-enhancement period, except for ECD and HOA RMS. Prior to the FDA approval of the TICL in 2018, patients with high degrees of myopic astigmatism had limited options for refractive correction. Reporting outcomes of bioptics in this patient population can be used as a benchmark for further studies on long-term outcomes of refractive surgery in high myopic astigmatism in the USA, including TICLs and small-incision lenticule extraction (SMILE).

One advantage of the two-step approach in high degrees of ametropia is the high refractive predictability. In this study, $85 \%$ of eyes achieved a MSE within $0.50 \mathrm{D}$ of target at one year, and this trend was stable at the last follow-up visit. The mean residual refractive target after ICL implantation $(-0.51 \pm 1.12 \mathrm{D})$ falls within a range that guarantees precise correction with an excimer-based approach. $^{18,19}$ In a retrospective comparative study between TICL implantation and bioptics for high myopic astigmatism, Alfonso et $\mathrm{al}^{20}$ demonstrated that bioptics were more likely to achieve MSE within 0.50 D (94\% vs $81 \%$ ) at 12-month postoperatively. Postoperative UDVA was also twice as likely to meet or exceed preoperative CDVA (57.8\% vs $32.1 \%$ ). Choi et $\mathrm{al}^{21}$ demonstrated no statistically significant difference between CDVA and UDVA at 12-month postoperatively between the TICL and bioptics group.
The introduction of wavefront-guided treatment and IR can ensure precise astigmatic correction, ${ }^{22-24}$ with most eyes achieving a non-visually significant astigmatism at the final postoperative visit $(0.34 \pm 0.40 \mathrm{D})$. Four enhancement treatments were performed without IR, with low postoperative residual astigmatism $(0.19 \pm 0.24 \mathrm{D})$, although the small number of eyes in this subgroup prevents any conclusive findings. In a previous study, residual refractive astigmatism was higher in the TICL group $(-0.42 \pm 0.32 \mathrm{D})$ than the bioptics group $(-0.32 \pm 0.38 \mathrm{D})$, with one eye requiring TICL rotation due to improper axis positioning. ${ }^{21}$

HOAs were found to increase at the 12 -month postoperative visit $(0.37 \pm 0.10 \mathrm{um})$, but this difference was not found to be statistically significant. Three patients complained of night glare following enhancement and were started on topical brimonidine with improvement in symptoms, which could be partly explained by an increase in HOAs. The study lacked contrast sensitivity assessment and subjective questionnaire about perceived visual quality. A previous study compared the optical and visual quality between TICL and bioptics using an adaptive optics visual simulator. ${ }^{25}$ TICL was found to provide better optical outcomes and contrast sensitivity than bioptics, especially when the pupil diameter was increased. Another study also demonstrated that ICL induce fewer ocular HOAs than wavefront-guided LASIK in moderate myopia. ${ }^{13}$ Nevertheless, the increase in $\mathrm{HOA}$ in this study remained acceptable, suggesting that an enhancement procedure could still guarantee very good visual outcomes following ICL implantation.

The main disadvantage of bioptics is the additive theoretical risks associated with two surgeries. The longer corneal wound healing associated with enhancement procedures could decrease the stability of the refraction over the first year. Our complication rate was low and comparable to the literature. ECL of $5.7 \%$ following ICL implantation was within the range of what has been previously reported. ${ }^{26,27}$ Endothelial cell counts were not routinely done following the 1-year follow-up except in the presence of corneal edema, which was not evident in any patient.

Since the arrival of the TICL, this approach has been abandoned at our center. The practicality, superior optical quality and reduced risk with TICL inarguably outweigh the advantages of bioptics with spherical ICL and would not warrant randomized controlled trials between both approaches. Regardless, excimer laser enhancement after phakic implants could still prevail as a valuable tool in the armamentarium of the refractive surgeon. 


\section{Funding}

There is no funding to report.

\section{Disclosure}

No conflicting relationships exist for any author.

\section{References}

1. Excimer laser photorefractive keratectomy (PRK) for myopia and astigmatism. American Academy of Ophthalmology. Ophthalmology. 1999;106(2):422-437.

2. Sugar A, Rapuano CJ, Culbertson WW, et al. Laser in situ keratomileusis for myopia and astigmatism: safety and efficacy: a report by the American Academy of Ophthalmology. Ophthalmology. 2002;109 (1):175-187. doi:10.1016/S0161-6420(01)00966-6

3. Schallhorn SC, Farjo AA, Huang D, et al. Wavefront-guided LASIK for the correction of primary myopia and astigmatism a report by the American Academy of Ophthalmology. Ophthalmology. 2008;115(7):1249-1261. doi:10.1016/j.ophtha.20 08.04.010

4. Hersh PS, Fry K, Blaker JW. Spherical aberration after laser in situ keratomileusis and photorefractive keratectomy. Clinical results and theoretical models of etiology. J Cataract Refract Surg. 2003;29 (11):2096-2104. doi:10.1016/j.jcrs.2003.09.008

5. Yoon G, Macrae S, Williams DR, Cox IG. Causes of spherical aberration induced by laser refractive surgery. $J$ Cataract Refract Surg. 2005;31(1):127-135. doi:10.1016/j.jcrs.2004.10.046

6. Sanders DR, Doney K, Poco M; ICL in Treatment of Myopia Study Group. United States Food and Drug Administration clinical trial of the implantable collamer lens (ICL) for moderate to high myopia: three-year follow-up. Ophthalmology. 2004;111(9): 1683-1692.

7. Alfonso JF, Baamonde B, Fernández-Vega L, Fernandes P, GonzálezMéijome JM, Montés-Micó R. Posterior chamber collagen copolymer phakic intraocular lenses to correct myopia: five-year follow-up. J Cataract Refract Surg. 2011;37(5):873-880. doi:10.1016/j.jcrs.20 10.11 .040

8. Huang D, Schallhorn SC, Sugar A, et al. Phakic intraocular lens implantation for the correction of myopia: a report by the American Academy of Ophthalmology. Ophthalmology. 2009;116(11):22 44-2258. doi:10.1016/j.ophtha.2009.08.018

9. Pineda-Fernández A, Jaramillo J, Vargas J, Jaramillo M, Jaramillo J, Galíndez A. Phakic posterior chamber intraocular lens for high myopia. J Cataract Refract Surg. 2004;30(11):2277-2283. doi:10.10 16/j.jcrs.2004.03.035

10. Zaldivar R, Davidorf JM, Oscherow S, Ricur G, Piezzi V. Combined posterior chamber phakic intraocular lens and laser in situ keratomileusis: bioptics for extreme myopia. J Refract Surg. 1999;15 (3):299-308.

11. Sánchez-Galeana CA, Smith RJ, Rodriguez X, Montes M, Chayet AS. Laser in situ keratomileusis and photorefractive keratectomy for residual refractive error after phakic intraocular lens implantation. J Refract Surg. 2001;17(3):299-304.

12. Arne JL, Lesueur LC, Hulin HH. Photorefractive keratectomy or laser in situ keratomileusis for residual refractive error after phakic intraocular lens implantation. J Cataract Refract Surg. 2003;29 (6):1167-1173. doi:10.1016/S0886-3350(03)00015-4
13. Kamiya K, Igarashi A, Shimizu K, Matsumura K, Komatsu M. Visual performance after posterior chamber phakic intraocular lens implantation and wavefront-guided laser in situ keratomileusis for low to moderate myopia. Am J Ophthalmol. 2012;153(6):1178-1186.

14. Kamiya K, Shimizu K, Aizawa D, Igarashi A, Komatsu M, Nakamura A. One-year follow-up of posterior chamber toric phakic intraocular lens implantation for moderate to high myopic astigmatism. Ophthalmology. 2010;117(12):2287-2294. doi:10.1016/j. ophtha.2010.03.054

15. Bhikoo R, Rayner S, Gray T. Toric implantable collamer lens for patients with moderate to severe myopic astigmatism: 12-month follow-up. Clin Exp Ophthalmol. 2010;38(5):467-474. doi:10.1111/ j.1442-9071.2010.02273.x

16. Sanders DR, Schneider D, Martin R, et al. Toric implantable collamer lens for moderate to high myopic astigmatism. Ophthalmology. 2007;114(1):54-61. doi:10.1016/j.ophtha.2006.08.049

17. Reinstein DZ, Archer TJ, Randleman JB. JRS standard for reporting astigmatism outcomes of refractive surgery. $J$ Refract Surg. 2014;30 (10):654-659. doi:10.3928/1081597X-20140903-01

18. Gomel N, Negari S, Frucht-Pery J, Wajnsztajn D, Strassman E, Solomon A. Predictive factors for efficacy and safety in refractive surgery for myopia. PLoS One. 2018;13(12):e0208608. doi:10.1371/ journal.pone.0208608

19. Wen D, Tu R, Flitcroft I, et al. Corneal surface ablation laser refractive surgery for the correction of myopia: a network meta-analysis. $J$ Refract Surg. 2018;34(11):726-735. doi:10.3928/1081597X-20180905-01

20. Alfonso JF, Lisa C, Fernández-Vega Cueto L, Fernandes P, GonzálezMéijome JM, Montés Micó R. Comparison of visual and refractive results of Toric implantable collamer lens with bioptics for myopic astigmatism. Graefes Arch Clin Exp Ophthalmol. 2013;251 (3):967-975. doi:10.1007/s00417-012-2155-9

21. Choi SH, Lee MO, Chung ES, Chung TY. Comparison of the toric implantable collamer lens and bioptics for myopic astigmatism. $J$ Refract Surg. 2011;27(2):91-97. doi:10.3928/1081597X-201004 14-01

22. Jun I, Kang DSY, Arba-Mosquera S, et al. Comparison between wavefront-optimized and corneal wavefront-guided transepithelial photorefractive keratectomy in moderate to high astigmatism. $B M C$ Ophthalmol. 2018;18(1):154. doi:10.1186/s12886-018-0827-x

23. Manche E, Roe J. Recent advances in wavefront-guided LASIK. Curr Opin Ophthalmol. 2018;29(4):286-291. doi:10.1097/ICU.0000 000000000488

24. Lee JJ, Kim MK, Wee WR. Effect of Iris registration on visual outcome in wavefront-guided LASEK for myopic astigmatism. Int Ophthalmol. 2018;38(2):513-525. doi:10.1007/s10792-017-04 86-6

25. Pérez-Vives C, Domínguez-Vicent A, Madrid-Costa D, FerrerBlasco T, Montés-Micó R. Myopic astigmatism correction: comparison of a Toric implantable collamer lens and a bioptics technique by an adaptive optics visual simulator. Ophthalmic Physiol Opt. 2013;33 (2):114-122. doi:10.1111/opo.12019

26. Shaaban YM, Badran TAF. Three-year effect of Phakic intraocular lenses on the corneal endothelial cell density. Clin Ophthalmol. 2020;14:149-155. doi:10.2147/OPTH.S236041

27. Nakamura T, Isogai N, Kojima T, Yoshida Y, Sugiyama Y. Posterior chamber Phakic intraocular lens implantation for the correction of myopia and myopic astigmatism: a retrospective 10-year follow-up study. Am J Ophthalmol. 2019;206:1-10. doi:10.1016/j.ajo.2019.04. 024 


\section{Publish your work in this journal}

Clinical Ophthalmology is an international, peer-reviewed journal covering all subspecialties within ophthalmology. Key topics include Optometry; Visual science; Pharmacology and drug therapy in eye diseases; Basic Sciences; Primary and Secondary eye care; Patient Safety and Quality of Care Improvements. This journal is indexed on PubMed

Submit your manuscript here: https://www.dovepress.com/clinical-ophthalmology-journal
Central and CAS, and is the official journal of The Society of Clinical Ophthalmology (SCO). The manuscript management system is completely online and includes a very quick and fair peer-review system, which is all easy to use. Visit http://www.dovepress.com/ testimonials.php to read real quotes from published authors. 